\title{
Resistance of Leishmania (Viannia) braziliensis to nitric oxide: correlation with antimony therapy and TNF- $\alpha$ production
}

Anselmo S Souza ${ }^{1 \dagger}$, Angela Giudice ${ }^{1 \dagger}$, Júlia MB Pereira', Luís H Guimarães ${ }^{1}$, Amelia R de Jesus², Tatiana R de Moura ${ }^{2}$, Mary E Wilson ${ }^{3 \dagger}$, Edgar M Carvalho ${ }^{1}$, Roque P Almeida ${ }^{2 *+}$

\begin{abstract}
Background: Nitric oxide (NO) produced in macrophages plays a pivotal role as a leishmanicidal agent. A previous study has demonstrated that $20 \%$ of the L. (V.) braziliensis isolated from initial cutaneous lesions of patients from the endemic area of Corte de Pedra, Bahia, Brazil, were NO resistant. Additionally, 5 to $11 \%$ of the patients did not respond to three or more antimony treatments" (refractory patients). The aim of this study is to investigate if there is an association between the resistance of $L$. (V.) braziliensis to $N O$ and nonresponsiveness to antimony therapy and cytokine production.
\end{abstract}

Methods: We evaluated the in vitro toxicity of $\mathrm{NO}$ against the promastigotes stages of $L$. (V.) braziliensis isolated from responsive and refractory patients, and the infectivity of the amastigote forms of these isolates against human macrophages. The supernatants from Leishmania infected macrophage were used to measure TNF- $\alpha$ and IL-10 levels.

Results: Using $\mathrm{NaNO}_{2}$ (pH 5.0) as the NO source, L. (V.) braziliensis isolated from refractory patients were more $\mathrm{NO}$ resistant (IC50 $=5.8 \pm 4.8)$ than $L$. (V.) braziliensis isolated from responsive patients (IC50 $=2.0 \pm 1.4$ ). Four isolates were selected to infect human macrophages: NO-susceptible and NO-resistant L. (V.) braziliensis isolated from responsive and refractory patients. NO-resistant $L$. (V.) braziliensis isolated from refractory patients infected more macrophages stimulated with LPS and IFN- $\gamma$ at 120 hours than NO-susceptible L. (V.) braziliensis isolated from refractory patients. Also, lower levels of TNF- $\alpha$ were detected in supernatants of macrophages infected with NO-resistant L. (V.) braziliensis as compared to macrophages infected with NO-susceptible L. (V.) braziliensis $(p<0.05$ at 2, 24 and 120 hours), while no differences were detected in IL-10 levels.

Conclusion: These data suggest that NO resistance could be related to the nonresponsiveness to antimony therapy seen in American Tegumentary Leishmaniasis.

\section{Background}

Leishmaniasis is a parasitic disease considered a major public health problem affecting 88 countries throughout Europe, Asia, Africa and America, with an annual incidence of 1 to 1.5 million of cases, and 350 million exposures according to the World Health Organization. Specifically in Brazil, the Ministry of Health reports an annual incidence of around 28.000 cases. American Tegumentary Leishmaniasis (ATL) presents with a spectrum of

\footnotetext{
* Correspondence: roquepacheco@uol.com.br

+ Contributed equally

${ }^{2}$ Universidade Federal de Sergipe, Aracaju, Sergipe, Brazil
}

clinical manifestations, including cutaneous $(\mathrm{CL})$, mucosal (ML), disseminated (DL) and diffuse cutaneous leishmaniasis (DCL). The major species that cause ATL in the New World are L. (Viannia) braziliensis, L. (V.) guyanensis, $L$. (Leishmania) amazonensis and $L$. (L.) mexicana [1]. In the Northeast region of Brazil, CL, ML and DL are caused most often by $L$. (V.) braziliensis; and DCL is most often caused by L. amazonensis in the New World [2]. The typical clinical manifestation of cutaneous leishmaniasis (CL) is a single ulcerated lesion with elevated borders, frequently located on the inferior limbs [3]. ML is a destructive disease that predominantly affects the 
nasopharynx. It is most common in the areas of $L$. (V.) braziliensis transmission and usually occurs months or years after CL [4]. DL is characterized by multiple pleomorphic acneiform, papular or nodular cutaneous lesions in two or more noncontiguous areas of the body [5].

Leishmania is a digenetic protozoa with two different life forms: promastigotes and amastigotes. Flagellated promastigotes replicate and mature to the infectious metacyclic form in the gut of sand flies. Promastigotes are transmitted to mammalian host by the bite of infected sand fly vector, which injects parasites into the host skin where they undergo facilitated phagocytosis by a macrophage and subsequently transform to obligate intracellular amastigotes [6,7]. Murine models have taught us that type 1 cell-mediated immune responses are key to immune protection against Leishmania spp. infections [8]. Interleukin (IL)-12 is an important cytokine that is produced by macrophages early in the infection. IL-12 induces interferon-gamma (IFN- $\gamma$ ) production by the Thelper $1\left(\mathrm{~T}_{\mathrm{H}} 1\right)$ and NK cells [9]. In murine systems, IFN- $\gamma$ has been shown to act in synergy with another macrophage derived cytokine, tumor necrosis factor alpha (TNF- $\alpha$ ), in activating macrophages and leading to the expression of inducible nitric oxide synthase (iNOS). iNOS catalyzes the synthesis of nitric oxide (NO) from arginine, a potent microbicidal agent that leads to killing of intracellular parasites and other microbes [10-12]. Our laboratory has described some $L$. (V.) braziliensis strains with resistance to NO Moreover, there is an association between the size of the initial cutaneous lesion of ATL and NO susceptibility of the parasite isolate in vitro. Furthermore, patients infected with NO-resistant $L$. braziliensis presented with larger cutaneous lesions than patients infected with NOsusceptible L. braziliensis [13].

The pentavalent antimonials $\left(\mathrm{Sb}^{\mathrm{V}}\right)$ meglumine antimoniate and stibogluconate have been the drugs of choice to treat leishmaniasis for decades [14]. The mechanism of $\mathrm{Sb}^{\mathrm{V}}$ action is unclear. A general consensus is that $\mathrm{Sb}^{\mathrm{V}}$ acts upon several targets in the parasite, including inhibition of parasite glycolysis, fatty acid beta-oxidation and ADP phosphorylation [15-17]. $\mathrm{Sb}^{\mathrm{V}}$ is most like reduced by the host cells to the trivalent form prior to its intracellular action $\left(\mathrm{Sb}^{\mathrm{III}}\right)$ [18]. L. infantum axenic amastigotes have been shown to undergo $\mathrm{Sb}^{\mathrm{III}}$ mediated DNA fragmentation, suggesting that apoptosis may participate in the mechanism of antimonial action [19]. Moreover, treatment of visceral leishmaniasis with pentavalent antimonials induces reactive oxygen species and NO generation in $L$. donovani-infected macrophages [20]. The emergence of antimony-resistant (SbR) visceral leishmaniasis (VL) in various parts of the world [21] has severely compromised the ability to control the disease. At the Corte de Pedra Health Post, located in an endemic area for cutaneous leishmaniasis situated in the southeast region of the state of Bahia, Brazil, the incidence of the disease is $8,1 / 1000$ habitants. A study defining phenotypic variations of parasite isolates at the point of clinical diagnosis could allow us to predict the evolution and the outcome of disease. Therefore, we evaluated phenotypic differences amongst $L$. (V.) braziliensis isolates from patients who did or did not respond to antimony therapy. Our data suggests that NO resistance could be related to the nonresponsiveness of American Tegumentary Leishmaniasis to antimony therapy.

\section{Methods}

Parasites and Patients recruiting

The patients described in this manuscript are from the endemic area of Corte de Pedra. Every 15 days our group travels to this population to treat leishmaniasis and other diseases. Patients are submitted for routine diagnostic evaluations that include parasite isolation and blood collection for serological purposes. All human subjects were briefed on procedures and signed informed consent documentation. All work with human subjects was carried out under Maternidade Climério de Oliveira Ethical Committee approval number 5/2006. $L$. $(V$.) braziliensis parasites were obtained by needle aspiration of the skin lesions in patients with ATL before therapy. Parasites were speciated by isoenzyme electrophoresis and monoclonal antibodies at the Departamento de Bioquímica e Biologia Molecular, Instituto Oswaldo Cruz, Fiocruz, Rio de Janeiro, Brazil [22].

\section{Responsive and refractory patients}

Subjects with ATL were identified and diagnosed at the Corte de Pedra Health Post, Bahia, Brazil. We analyzed 1640 cutaneous leishmnaisis cases from Corte de Pedra, between 2001 and 2003, and found that 5\% of the patients needed 3 courses of antimony treatment to heal their lesions, and $11 \%$ of the CL cases were refractory to more than 3 courses of antimony treatment. For this present study, patients who healed their lesions with one course of antimony therapy $(20 \mathrm{mg} \mathrm{Sb} / \mathrm{kg} /$ day during twenty days) were considered responsive patients. Patients who received three or more courses of antimony therapy were considered refractory patients. The interval between the courses of treatment was 60 days. Sixteen parasite isolates were studied in total: eight were isolated from responsive patients (Table 1) and eight were isolated from refractory patients (Table 2).

\section{Isolation and cultivation of $L$. (V.) braziliensis}

Parasites isolates $L$. (V.) braziliensis were initially cultivated in tubes with biphasic medium (NNN) consisting of rabbit blood agar overlaid with liver infusion tryptose 
Table 1 Optical density of $L$. (V.) braziliensis isolated from responsive patients to antimony therapy exposed to $\mathrm{NaNO}_{2}$

\begin{tabular}{lllllllll}
\hline Isolates & & \multicolumn{8}{c}{$\mathrm{NaNO}_{\mathbf{2}}(\mathbf{m M})$} \\
& $\mathbf{0}$ & $\mathbf{0 . 2 5}$ & $\mathbf{0 . 5}$ & $\mathbf{1}$ & $\mathbf{2}$ & $\mathbf{4}$ & $\mathbf{8}$ & $\mathbf{1 6}$ \\
\hline 14048 & 0.401 & 0.194 & 0.214 & 0.206 & 0.095 & 0.086 & 0.071 & 0.089 \\
14207 & 0.397 & 0.361 & 0.330 & 0.350 & 0.242 & 0.106 & 0.094 & 0.082 \\
14806 & 0.301 & 0.379 & 0.339 & 0.186 & 0.086 & 0.044 & 0.019 & 0.005 \\
$\mathbf{1 5 1 7 1}$ & $\mathbf{0 . 4 0 1}$ & $\mathbf{0 . 3 1 6}$ & $\mathbf{0 . 2 3 8}$ & $\mathbf{0 . 1 6 5}$ & $\mathbf{0 . 0 8 8}$ & $\mathbf{0 . 0 5 6}$ & $\mathbf{0 . 0 4 0}$ & $\mathbf{0 . 0 4 5}$ \\
$\mathbf{1 5 4 0 4}$ & $\mathbf{0 . 5 8 5}$ & $\mathbf{0 . 4 5 9}$ & $\mathbf{0 . 3 5 3}$ & $\mathbf{0 . 3 8 5}$ & $\mathbf{0 . 3 8 3}$ & $\mathbf{0 . 2 0 8}$ & $\mathbf{0 . 1 7 4}$ & $\mathbf{0 . 1 7 8}$ \\
15468 & 0.493 & 0.312 & 0.232 & 0.241 & 0.259 & 0.109 & 0.085 & 0.063 \\
15492 & 0.396 & 0.321 & 0.237 & 0.228 & 0.098 & 0.071 & 0.047 & 0.059 \\
15826 & 0.296 & 0.125 & 0.149 & 0.128 & 0.120 & 0.084 & 0.081 & 0.082
\end{tabular}

Values represent means from three experiments for each parasite isolate. Bold face is used to mark the isolates chosen for further studies, illustrated in Figure 2.

(LIT), supplemented with $10 \%$ heat inactivated fetal bovine serum medium (Sigma Chemical Co., St. Louis, $\mathrm{MO})$. Following isolation, these parasites were cryopreserved. After selection for the present study, parasite cultures were expanded in Schneider's insect medium (Sigma) pH 7.2 supplemented with $10 \%$ fetal bovine serum (FBS) and $2 \%$ human male urine at $25^{\circ} \mathrm{C}$ (complete Schneider medium).

\section{Evaluation of NO susceptibility of $L$. (V.) braziliensis promastigotes by MTT assay}

The virulence of Leishmania spp. is highest in the stationary phase, a growth phase that is enriched for the infectious metacyclic stage of the organism. The sensitivity of MTT uptake assays, however, is best using log phase parasites. We previously reported that MTT assays of oxidant sensitivity of log phase cultures correlates with oxidant sensitivity and virulence of the same isolate when it reaches stationary phase growth $[23,24]$.

Two hundred $\mu \mathrm{L}$ of $L$. (V.) braziliensis promastigotes isolated from responsive $(n=8)$ or refractory patients

Table 2 Optical density of $L$. (V.) braziliensis isolated from refractory patients to antimony therapy exposed to $\mathrm{NaNO}_{2}$

\begin{tabular}{lllllllll}
\hline Isolates & \multicolumn{8}{c}{$\mathrm{NaNO}_{\mathbf{2}}(\mathbf{m M})$} \\
& $\mathbf{0}$ & $\mathbf{0 . 2 5}$ & $\mathbf{0 . 5}$ & $\mathbf{1}$ & $\mathbf{2}$ & $\mathbf{4}$ & $\mathbf{8}$ & $\mathbf{1 6}$ \\
\hline $\mathbf{3 9 3}$ & $\mathbf{0 . 5 3 1}$ & $\mathbf{0 . 5 3 4}$ & $\mathbf{0 . 5 8 5}$ & $\mathbf{0 . 6 1 4}$ & $\mathbf{0 . 5 3 6}$ & $\mathbf{0 . 4 5 8}$ & $\mathbf{0 . 3 6 6}$ & $\mathbf{0 . 2 8 3}$ \\
14119 & 0.474 & 0.354 & 0.387 & 0.358 & 0.230 & 0.163 & 0.114 & 0.121 \\
14758 & 0.436 & 0.367 & 0.355 & 0.389 & 0.353 & 0.228 & 0.145 & 0.138 \\
14808 & 0.451 & 0.447 & 0.517 & 0.408 & 0.341 & 0.227 & 0.171 & 0.138 \\
14933 & 0.475 & 0.428 & 0.437 & 0.353 & 0.192 & 0.168 & 0.135 & 0.154 \\
15028 & 0.420 & 0.294 & 0.383 & 0.415 & 0.244 & 0.122 & 0.120 & 0.104 \\
15226 & 0.409 & 0.412 & 0.370 & 0.432 & 0.327 & 0.223 & 0.133 & 0.138 \\
$\mathbf{1 5 3 4 4}$ & $\mathbf{0 . 3 8 2}$ & $\mathbf{0 . 2 3 2}$ & $\mathbf{0 . 1 7 1}$ & $\mathbf{0 . 2 4 9}$ & $\mathbf{0 . 1 8 3}$ & $\mathbf{0 . 0 7 8}$ & $\mathbf{0 . 0 5 3}$ & $\mathbf{0 . 0 5 6}$
\end{tabular}

Values represent means from three experiments for each isolate. Bold face is used to mark the isolates chosen for further studies, illustrated in Figure 3. $(\mathrm{n}=8)$ in $\log$ phase growth at $2.5 \times 10^{7}$ parasites $/ \mathrm{mL}$ in Hanks' balanced solution (HBSS, Sigma, pH 5.0) were exposed to dilutions of $\mathrm{NaNO}_{2}$ (NO donor) from 0 to $16 \mathrm{mM}$. After 4 hours at $25^{\circ} \mathrm{C}$, plates were centrifuged and parasites were incubated with $5 \mathrm{mg}$ of $\mathrm{MTT} / \mathrm{mL}$ [3-(4,5-dimethylthyazol)-2,5-diphenyltetiazoliumbromide] at $25^{\circ} \mathrm{C}$ for 4 hours. Reactions were stopped with $0.04 \mathrm{~N} \mathrm{HCl}$ in isopropanol and conversion of yellow MTT to purple formazan, indicating mitochondrial metabolism, was detectable at $540 \mathrm{~nm}$. The percentage of viability was calculated by comparison to MTT concentrations in wells without $\mathrm{NaNO}_{2}$ [23]. At least 3 separate NO toxicity assays were performed on each parasite isolate.

\section{Macrophages culture}

Peripheral blood mononuclear cells (PBMCs) were isolated from six different healthy human donors. Briefly, heparinized blood was diluted $1: 2$ with $0.15 \mathrm{M} \mathrm{NaCl}$, and separated on a Ficoll Hypaque gradient (LSM; Organon Teknika corporation, Durham, NC, USA). Mononuclear cells were collected from the interface between the plasma and the Ficoll. Washed mononuclear cells were suspended in RPMI 1640, 10\% heat inactivated AB serum (Sigma), penicillin/streptomycin (complete medium) (Gibco BRL, Grant Island, NY). One $\times 10^{6}$ cells in $200 \mu \mathrm{L}$ allowed to adhere to 8-well Lab Tek plates for 2 hours at $37^{\circ} \mathrm{C}, 5 \% \mathrm{CO}_{2}$. Non-adherent cells were removed by rinsing, and the resultant adherent monocytes were allowed to differentiate to monocyte-derived macrophages over six days at $37^{\circ} \mathrm{C}$ in $5 \% \mathrm{CO}_{2}$.

\section{Macrophage infections}

Macrophages were infected by using four of the above described L. (V.) braziliensis promastigotes in the stationary growth phase, 2 isolated from antimony responsive patients (LTCP 15171 and 15404) and 2 isolated from antimony refractory patients (LTCP 393 and 15344). Growth curves of these L. braziliensis isolates were performed and no statistically significant differences were observed between the isolates used for macrophage infection. Promastigotes were grown to the stationary phase, washed by centrifugation, suspended in RPMI 1640 and used to infect macrophages from healthy donors. In each experiment, the macrophages were infected with NO susceptible and NO resistant parasites. Care was taken to infect macrophages from the same individual with NO-resistant and NO-susceptible strains. The infections were done at a parasite/ macrophage ratio of $5: 1$ for 2 hours at $35^{\circ} \mathrm{C}$ in $5 \% \mathrm{CO}_{2}$. Extracellular parasites were then removed by gentle washing, and infected macrophages were maintained for an additional 2, 24, 72 and 120 hours. Conditions 
did or did not contain LPS (100 ng/mL) plus IFN- $\gamma$ $(10 \mathrm{ng} / \mathrm{mL})$, added at thirty minutes before the infection. Cells were stained with Giemsa and the infection levels were enumerated microscopically. Each isolate was tested in at least three separate assays. Macrophages from the six healthy donors were used for the infection assays by all the selected isolates. Although the number of parasite samples selected for macrophage infections is small, these data clearly characterize the differences in the behavior between the parasites with different to NO susceptibilities previously described by our group [13]. The proportion of infected cells and the number of parasite/100 macrophages were enumerated by three independent observers, blinded to the experimental conditions.

\section{Cytokine determination}

The supernatants from cultures were harvested at 2, 24, 72 and 120 hours post infection and stored at $-20^{\circ} \mathrm{C}$ for TNF- $\alpha$ and IL-10 determination by sandwich ELISA technique (TNF- $\alpha$ : R\&D Systems, Minneapolis, MN; IL10: BD-Pharmingen). A standard curve was generated using recombinant TNF- $\alpha$ and recombinant IL-10 to express the results in $\mathrm{pg} / \mathrm{mL}$.

\section{Statistical analysis}

Mann-Whitney test was used to compare susceptibility/ resistance to NO between $L$. (V.) braziliensis isolates.

One-way ANOVA with Tukey's test to compare the levels of cytokines released from infected macrophages.

Kruskal-Wallis test, Dunn's multiple comparison Test was used to compare the means obtained from infection and multiplication of $L$. (V.) braziliensis in human macrophages.

The analyses were done on GraphPad Prism 3.03 software (San Diego, CA, USA).

A p value $<0.05$ was considered as the cutoff for statistical significance.

\section{Results}

\section{L. (V.) braziliensis isolated from refractory patients were} more resistant to NO than $L$. (V.) braziliensis isolated from responsive patients

We compared the resistance to NO against the responsiveness to antimony therapy of $L$. $(V$.) braziliensis isolates from patients. We investigated the NO toxicity by measuring the viability of log phase organisms exposed to a gradient of the NO-generating agent $\mathrm{NaNO}_{2}$. Viability was measured according to the ability of the parasites to convert MTT to formazan, a measure of mitochondrial activity (Tables 1 and 2). L. (V.) braziliensis isolated from antimony-refractory patients were more resistant to $\mathrm{NO}$ in vitro than $L$. (V.) braziliensis isolated from antimonyresponsive patients. The average IC50 (50\% inhibitory

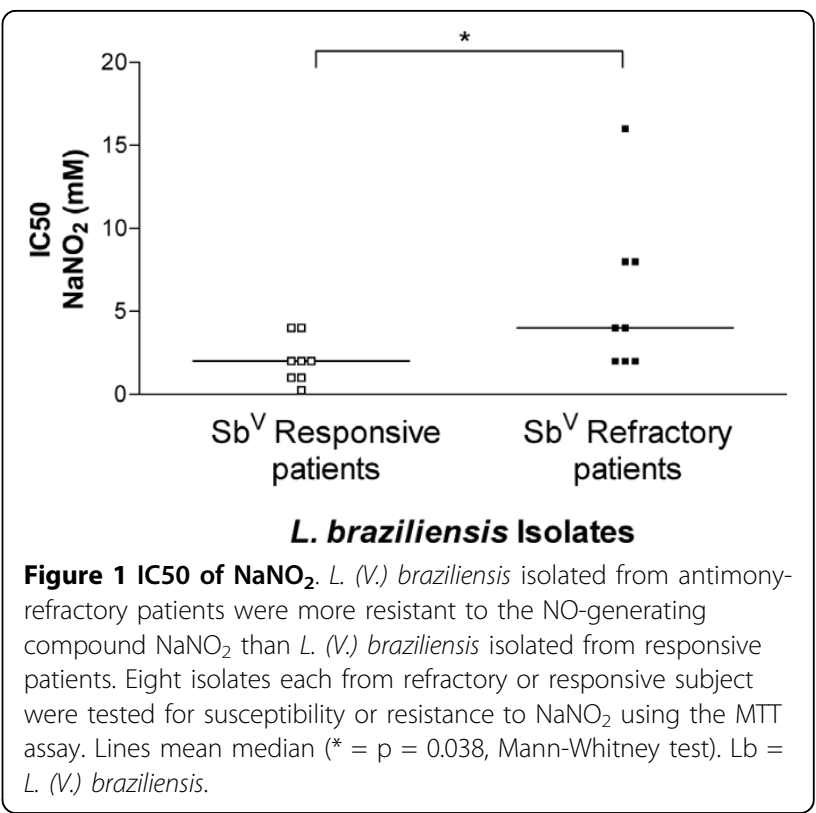

concentration) was $2.0 \pm 1.4 \mathrm{mM} \mathrm{NaNO}_{2}$ for isolates from susceptible, compared to $5.8 \pm 4.8 \mathrm{mM} \mathrm{NaNO}_{2}$ for isolates from resistant subjects (Figure 1). Defining $4 \mathrm{mM} \mathrm{NaNO}_{2}$ as intermediate and $>4 \mathrm{mM} \mathrm{NaNO}_{2}$ as resistant, only 2 of the 8 isolates from antimony-responsive subjects were intermediate and 6 of the 8 were susceptible. This is in contrast to isolates from antimony-resistant subjects, amongst which 5 of the 8 isolates were either intermediate or resistant and only 3 of 8 isolates were susceptible. These data suggest that NO-resistance might be associated with some cases of resistance to antimony therapy.

\section{Infection of human macrophages with $L$. (V.) braziliensis isolates from patients that were responsive or refractory to antimony therapy}

To assess whether macrophage infection was influenced by NO resistance and/or antimony responsiveness, we selected four of the above isolates, one susceptible and one intermediate-to-resistant each from the antimonyresponsive and the antimony-refractory groups [LTCP 15344 (NO-susceptible) and LTCP 393 (NO-resistant) isolated from refractory patients; LTCP 15171 (NO-susceptible) and LTCP 15404 (NO-resistant) isolated from responsive patients]. Parasites were used to infect human monocyte-derived macrophages (MDMs) from three different healthy donors. After 2, 24, 72 or 120 hours of infection the parasite load was quantified microscopically to assess entry and survival in macrophages. Data using isolates from antimony-responsive patients are shown in Figure 2. After 24 hours of infection, NO-susceptible $L$. (V.) braziliensis infected more macrophages than the NO-resistant parasites 

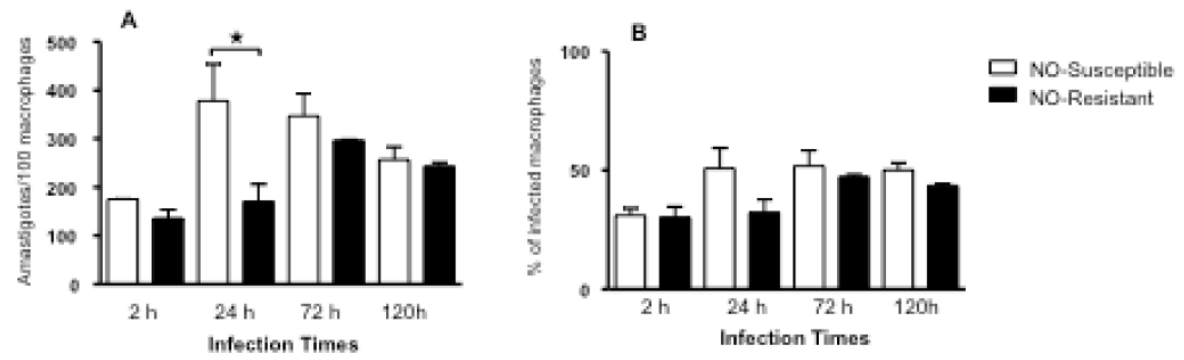

IFN- $\gamma$ and LPS treated Macrophages
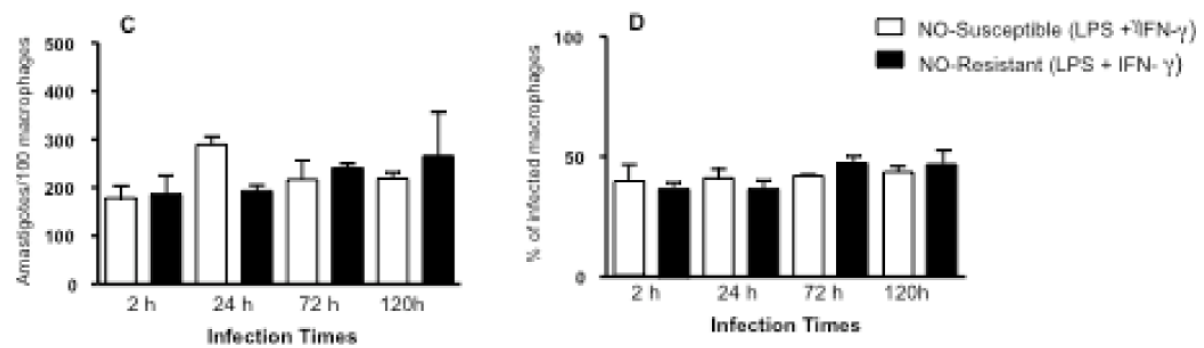

Figure 2 Infection of human macrophages with L. (V.) braziliensis (NO-susceptible - open bars and NO-resistant - closed bars) isolated from patients who responded to antimony therapy. $A$ and $B$, Monocyte-derived macrophages were infected with $L$. (V.) braziliensis promastigotes (5:1 ratio). At 2, 24, 72 and $120 \mathrm{~h}$ of culture(A) the number of intracellular amastigotes and (B) the percentage of infected cells was quantified microscopically as described in Methods. (C) and (D) Monocyte-derived macrophages were treated with LPS (100 ng/mL) plus IFN- $\gamma(10 \mathrm{ng} / \mathrm{mL})$ thirty minutes before infection with L. (V.) braziliensis promastigotes. At 2, 24, 72 and $120 \mathrm{~h}$ of culture, (C) the number of intracellular amastigotes and $(D)$ the percentage of infected cells was quantified microscopically. Each bar represents the mean \pm SEM infection level in MDMs from three donors (Kruskal-Wallis test, Dunn's multiple comparison Test, ${ }^{*}=p<0.05$ ).

(Figure 2A). However, at 120 hours the two isolates had similar levels of infection. When expressed as the percentage of infected macrophages, there was a trend toward increased infection by NO-susceptible $L$. $(V$.$) braziliensis which did not reach statistical signifi-$ cance (Figure 2B). To evaluate whether NO resistance is manifested by resistance to macrophage leishmanicidal activity, we infected macrophages with $L$. $(V$.) braziliensis isolated from responsive patients after LPS and IFN- $\gamma$ (Figure 2C and 2D). There was little difference between the infection levels at any time point, suggesting that NO-resistance or susceptibility does not make these $L$. $(V$.) braziliensis isolates responsive to leishmanicidal activity by MDMs exposed to strong activators of classical activation.

MDM infection with $L$. (V.) braziliensis isolated from antimony-refractory patients showed similar results. The NO-susceptible parasites infected more macrophages at 24 hours of infection compared to the NO-resistant parasites, although the difference was not statistically significant (Figure 3A-B). In contrast to isolates from antimony-responsive subjects, after 120 hours of infection NO-resistant $L$. (V.) braziliensis from antimony-refractory patients achieved a significantly higher burden of infection than NO-susceptible isolates (53 \pm 14 amastigotes/100 macrophages). The addition of LPS plus IFN- $\gamma$ did not affect the results (Figures 3C-D). Overall these results indicate that NO-susceptible isolates achieve a high level of initial infection but are rapidly killed over 120 hrs, whereas NO-resistant isolates either maintain the original infection level (Figure 2) or increase infection (Figure 3).

TNF- $\alpha$ production is not induced in macrophages infected with NO-resistant $L$. $(V$.$) braziliensis$

TNF- $\alpha$ is a proinflammatory cytokine important for the intracellular control of Leishmania infection [11]. We evaluated whether TNF- $\alpha$ production is impaired during infection with NO-resistant (LTCP 393 and LTCP 15404) compared to NO-susceptible (LTCP 15171 and LTCP 15344) L. (V.) braziliensis isolates from antimonyrefractory or antimony-responsive patients. TNF- $\alpha$ levels were measured in supernatants from infected macrophages cultures. 


\section{Untreated Macrophages}
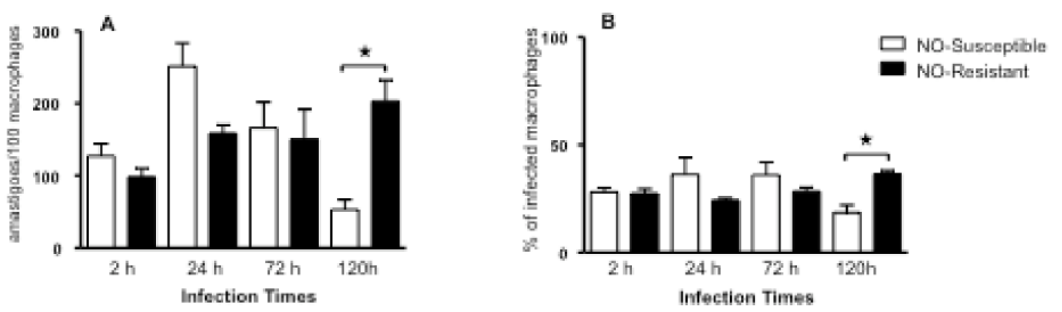

IFN- $\gamma$ and LPS treated Macrophages
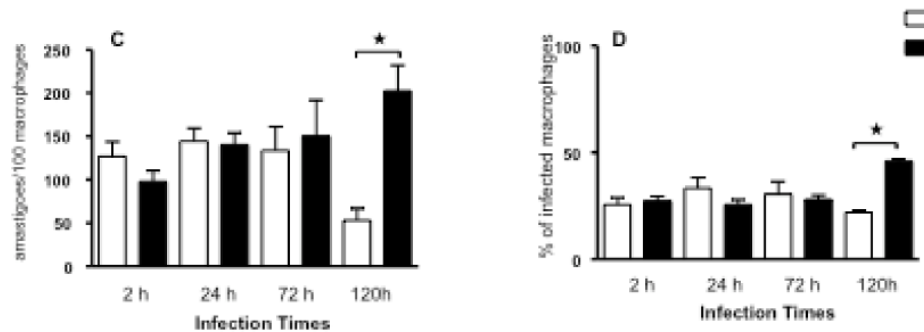

Figure 3 Infection of human macrophages with L. (V.) braziliensis (NO-susceptible - open bars and NO-resistant-closed bars) isolated from patients who were refractory to antimony therapy. $(A)$ and $(B)$ Monocyte-derived macrophages were infected with $L$. (V.) braziliensis promastigotes (5:1 ratio). At 2, 24, 72 and $120 \mathrm{~h}$ of culture the number of intracellular amastigotes(A) and the percentage of infected cells (B) was quantified microscopically as described in Methods. (C) and (D) Monocyte-derived macrophages were treated with LPS (100 ng/mL) plus IFN- $\gamma(10 \mathrm{ng} / \mathrm{mL})$ thirty minutes before the infection with L. (V.) braziliensis promastigotes. At 2, 24, 72 and $120 \mathrm{~h}$ of culture, (C) the number of intracellular amastigotes and $(D)$ the percentage of infected cells was quantified microscopically. Each bar represents the mean \pm SEM parasite loads in MDMs from three donors (Kruskal-Wallis test, Dunn's multiple comparison Test, ${ }^{*}=p<0.05$ ).

Non-stimulated macrophages did not produce TNF- $\alpha$ at a level that differed significantly from medium when they were infected with NO-susceptible and NO-resistant L. (V.) braziliensis. The positive control stimulation, LPS + IFN- $\gamma$, led to a significant increase of TNF- $\alpha$ production at all time points (Figure 4A). However, macrophages that were stimulated with LPS and IFN- $\gamma 30 \mathrm{~min}$ prior to infection with $\mathrm{NO}$-susceptible $L$. (V.) braziliensis produced significantly more TNF- $\alpha$ at all time points tested ( 2 h: $1954 \pm 397 ; 24$ h: $5101 \pm 424 ; 72$ h: $4766 \pm$ 260; 120 h: $5407 \pm 141$ ), when compared to macrophages infected with NO-resistant isolates ( $2 \mathrm{~h}: 804 \pm 193$; 24 h: $1986 \pm 347 ; 72$ h: $2233 \pm 842 ; 120$ h: $1244 \pm 252)$ or uninfected control (Figure 4B). The results are expressed as mean \pm standard error of mean in $\mathrm{pg} / \mathrm{mL}$. These data suggest that NO-resistant $L$. (V.) braziliensis do not induce TNF- $\alpha$ production by macrophages whereas $\mathrm{NO}$-susceptible isolates induces significant TNF- $\alpha$ production.

\section{IL-10 production is not altered in macrophages infected} with $L(V)$ braziliensis isolated from refractory patients IL-10 is a cytokine that suppresses NO-production in macrophages. We therefore inquired whether the NOresistant isolates suppressed TNF- $\alpha$ through enhanced IL-10 production. There were no statistically significant differences in IL-10 production in non-stimulated macrophages (Figure 5A). There was a trend toward increased IL-10 in the presence of LPS + IFN- $\gamma 72 \mathrm{hrs}$ after stimulation $(11 \pm 5 \mathrm{pg} / \mathrm{mL})$, suggesting a secondary compensatory increase as has been observed in prior reports [25]. Paradoxically, in the constant presence of LPS + IFN- $\gamma$, macrophages infected with NO-susceptible $L$. $(V$.) braziliensis produced higher levels of IL-10 (24 h: $86 \pm 48 ; 72$ h: $52 \pm 40 ; 120$ h: $21 \pm 19$ ) than non-infected macrophages ( $1 \mathrm{pg} / \mathrm{mL}$ in all time points) or macrophages infected with NO-resistant L. (V.) braziliensis (approximately $1 \mathrm{pg} / \mathrm{mL}$ in all time points). However, statistically significant differences were not found (Figure 5B). These data suggest it is likely that another suppressor mechanism is responsible for the enhanced growth of NO-resistant parasites isolated from antimony refractory patients. It seems that these parasites do not activate macrophage to produce either TNF- $\alpha$ or IL-10, while NO-susceptible induce both cytokines.

\section{Discussion}

Nitric oxide (NO) generated through the activity of the inducible nitric oxide synthase (iNOS) is a critical component of intracellular killing of Leishmania in murine macrophages and clearance of disease in mice [26,27]. More recently, the importance of NO in the microbial 


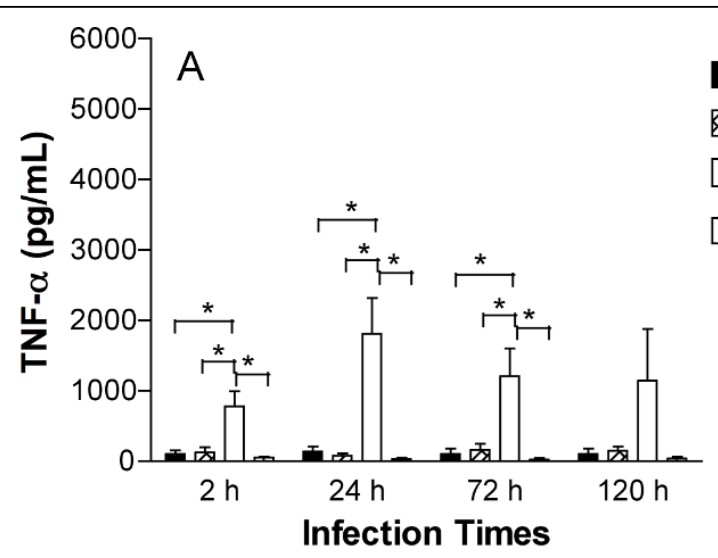

NO-Resistant NOM-Susceptible

LPS + IFN- $\gamma$

m. Medium

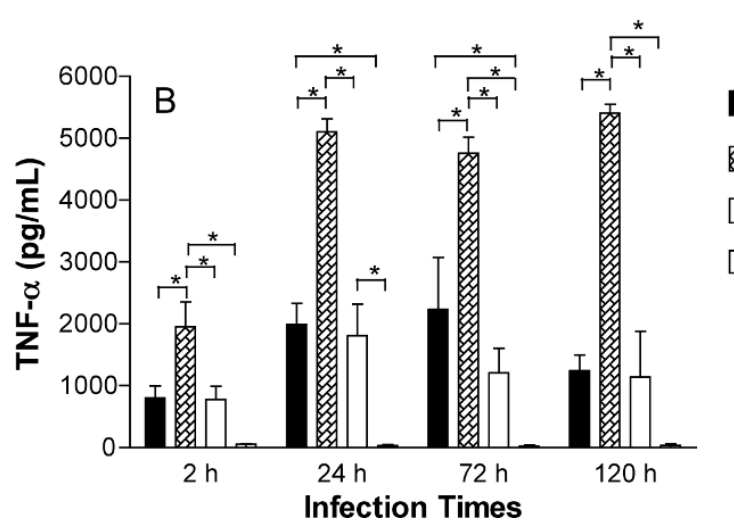

Figure 4 TNF- $\alpha$ production is impaired in macrophages infected with NO-resistant $L$. (V.) braziliensis. Monocyte-derived macrophages were infected with NO-susceptible or NO-resistant L. (V.) braziliensis. After 2, 24, 72 or $120 \mathrm{~h}$ of infection the culture supernatants were harvest from non-stimulated macrophages (A) or macrophages treated with LPS $(100 \mathrm{ng} / \mathrm{mL})$ plus IFN- $\gamma(10 \mathrm{ng} / \mathrm{mL})$ thirty minutes prior to the addition of parasites (B). TNF- $\alpha$ levels were determined by ELISA. Each bar represents the mean \pm SEM of three experiment from three separate donors (ANOVA, Tukey's multiple comparison Test, ${ }^{*}=p<0.05$ ).

defenses of human macrophages against both Leishmania spp. and Mycobacterium tuberculosis has been suggested $[28,29]$. Recent data from our group demonstrated that NO resistance of human $L$. $(V$. $)$ braziliensis isolates correlates directly with the initial size of the cutaneous lesion. Thus, patients infected with NO-resistant Leishmania present with a larger initial lesion size than patients infected with NO-resistant Leishmania [13]. L. (V.) braziliensis is a cause of cutaneous, mucosal and disseminated cutaneous leishmaniasis in endemic regions. Although some of these cutaneous lesions respond immediately to antimony treatment, other infections can be difficult to cure and require multiple courses of therapy [30,31].

We hypothesized that there may be inherent biological differences between the parasites isolates causing antimony-responsive versus antimony-refractory cutaneous lesions. The purpose of the current study was to evaluate phenotypic differences between $L$. (V.) braziliensis isolates from patients with localized cutaneous leishmaniasis who did or did not respond to antimony therapy.

Sensitivity of $L$. (V.) braziliensis parasite isolates to nitric oxide was measured using the MTT assay as a measure of viability after exposure to acidified $\mathrm{NaNO}_{2}$, a compound that releases $\mathrm{NO}$ and induces leishmanicidal activity [32]. We observed that more of the $L$. (V.) braziliensis isolates from antimony-refractory patients were NO resistant than $L$. (V.) braziliensis isolates derived from antimony-responsive patients. Furthermore, all the highly NO-resistant isolates were obtained from antimony-refractory patients.

Evaluation of human macrophage infections with NOsusceptible or NO-resistant isolates from either antimony-responsive or antimony-refractory patients also demonstrated that there are biological differences between the isolates, which correlate with NO susceptibility. NO-susceptible parasites exhibited transient 

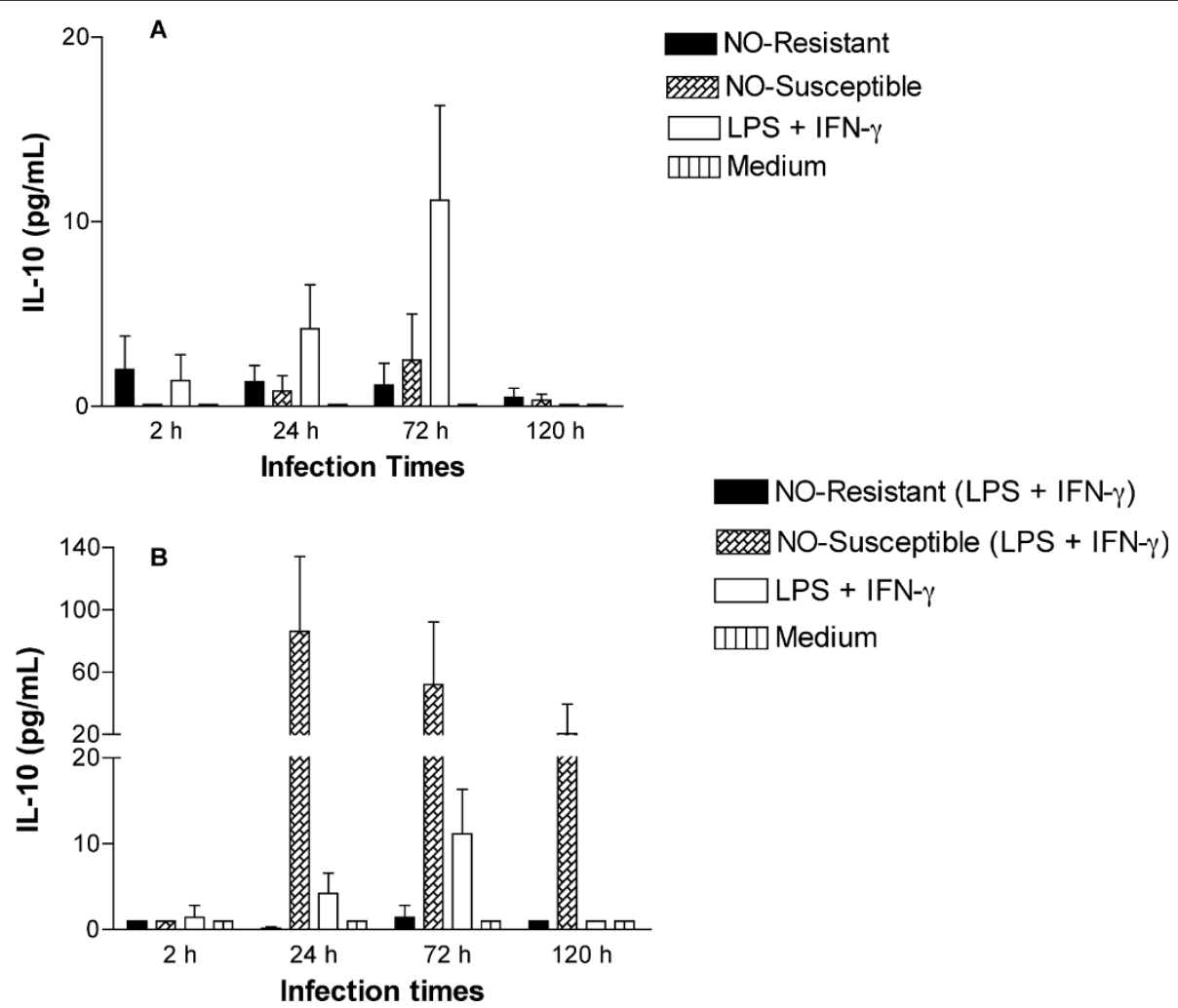

Figure 5 IL-10 production not is altered in macrophages infected with isolated from refractory patients. Monocyte-derived macrophages were infected with NO-susceptible and NO-resistant L. (V.) braziliensis. After 2, 24, 72 or $120 \mathrm{~h}$ of infection the culture supernatants of unstimulated macrophages (A) or from macrophages treated with LPS $(100 \mathrm{ng} / \mathrm{mL})$ plus IFN- $\gamma(10 \mathrm{ng} / \mathrm{mL})$ for thirty minutes before infection (B), were assessed for IL-10 levels by ELISA. Each bar represents the mean \pm SEM infection levels in MDMs from three separate donors.

initial growth in macrophages followed by intracellular killing, but only with a significant difference in NO-sensitive isolate from an antimony-responsive patient. The NO-resistant $L$. (V.) braziliensis isolates tested herein did not exhibit the initial growth phase or intracellular killing. Indeed, the highly NO-resistant isolate from an antimony-refractory patient displayed significant intracellular growth by $120 \mathrm{hrs}$ of infection, even in the presence of LPS and IFN- $\gamma$, compounds that usually activate macrophages to become leishmanicidal [26,33]. The fact that both NO-susceptible and NO-resistant L. (V.) braziliensis isolates were maintained out to 120 hours of macrophage infection, even in the presence of LPS and IFN- $\gamma$ (Figure 2), suggests that isolates can be resistant to other leishmanicidal mechanisms, including products of the NADPH oxidase (hydrogen peroxide, superoxide) [34-36].

It was previously reported that an isolate of $L$. infantum resistant to trivalent antimony $\left(\mathrm{Sb}^{\mathrm{III}}\right)$ in vitro was also resistant to an exogenous NO donor and to intracellular killing in activated macrophages [18]. Pentavalent antimony $\left(\mathrm{Sb}^{\mathrm{V}}\right)$ must be reduced to $\mathrm{Sb}^{\mathrm{III}}$ to exhibit its leishmanicidal activity $[18,19] . \mathrm{Sb}^{\mathrm{III}}$ in turn induces the production of reactive oxygen and nitrogen radicals in macrophages infected with $L$. donovani [20]. It follows logically that there may be a direct association between non-responsiveness to antimony therapy and NO resistance. Whether these isolates are also resistant to hydrogen peroxide and superoxide must still be tested. It has been demonstrated in Leishmania donovani that the parasite uses a cascade of enzymes that include cytosolic tryparedoxin peroxidase (cTXNPx) for the detoxification of peroxides, a mechanism necessary for the survival of digenetic parasites living in two disparate biological environments. The exposure of the parasite to a combination of $\mathrm{H}_{2} \mathrm{O}_{2}$ and nitric oxide resulted in a significant reduction of cTXNPx levels accompanied by high cell death. However, overexpression of cTXNPx by transfection increased the virulence, parasite burden in macrophages and resistance to clearance by antimony therapy, suggesting that differential expression of cTXNPx is linked to parasite resistance and virulence. [37].

The ability of $L$. (V.) braziliensis isolates to sustain infection may be related to their ability to resist $\mathrm{NO}$ toxicity [38]. Since arginine is a substrate of both iNOS 
and arginase, and the amount of arginine substrate is rate-limiting for both enzymes, consumption of arginine by the parasite can alter the activity of either enzyme in the intracellular environment. Arginase-mediated L-arginine hydrolysis produces polyamines which promote the growth of Leishmania [39,40], whereas arginine metabolism through NOS leads to toxic NO production. L. amazonensis produce nitric oxide synthase (NOS), at especially high levels in the axenic amastigote stage [41], and all Leishmania species studied make arginase [40]. The presence of either enzyme could deplete environmental arginine, generating either compartmentalized NO not available to kill Leishmania or generating polyamines that stimulate parasite growth [42]. Overactivity of either parasite enzyme would sequester arginine away from host. In addition to suppressing its synthesis through scavenging substrate, Leishmania may inhibit NO generation indirectly by inhibiting the macrophage synthesis of IL-12, decreasing the amount of IFN- $\gamma$ produced by $\mathrm{T}$ and other cells, and in turn down-regulating the amount of NO produced through classical macrophage activation [43].

Using supernatants from culture of macrophages infected with NO-susceptible and NO-resistant $L$. (V.) braziliensis, we determined that macrophages stimulated with LPS and IFN- $\gamma$ produced significantly lower amounts of TNF- $\alpha$ when they were infected with NO-resistant $L .(V$.) braziliensis than when they were infected with NO-susceptible $L$. $(V$.) braziliensis at all time points measured. This result suggests that NO-resistant $L .(V$.$) braziliensis do not induce TNF- \alpha$ production by macrophages.

TNF- $\alpha$ is a proinflammatory cytokine produced by macrophages and lymphocytes and it is important in the control of Leishmania spp. infections [11]. The impairment of TNF- $\alpha$ production by NO-resistant $L$. (V.) braziliensis is consistent with the finding that these isolates are present in patients with a severe form of tegumentary leishmaniasis [44].

Leishmania parasites can induce the production and/or secretion of various immunosuppressive signaling molecules including arachidonic acid metabolites and the cytokines TGF- $\beta$ and IL-10 [45]. IL-10 in turn suppresses IFN- $\gamma$ and TNF- $\alpha$, and consequently inhibits NO-production by macrophages [46]. As a first step in examining mechanisms of TNF- $\alpha$ suppression, we examined whether IL-10 was induced by NO-resistant L. (V.) braziliensis. Disproving our hypothesis, we observed minimal IL-10 production by non-stimulated macrophages infected with NO-susceptible and NOresistant $L$. (V.) braziliensis. Paradoxically, when macrophages were stimulated with LPS and IFN- $\gamma$ and infected with NO-susceptible $L$. $(V$.) braziliensis they produced high levels of IL-10, whereas macrophages infected with NO-resistant $L$. (V.) braziliensis produced minimal. We interpret this as a compensatory increase in IL-10 in response to the increased type 1 cytokines induced, illustrated by the TNF- $\alpha$ increase observed in Figure 4. These results show that enhanced IL-10 is not the mechanism leading to absence of TNF- $\alpha$ production in macrophages infected with NO-resistant $L$. (V.) braziliensis. It is likely that another mechanism generates the immunosuppressive signal delivered by the resistant parasites. Candidates would include PGE2, which favors parasite intracellular survival by suppressing TNF- $\alpha$, IL-1 and reactive oxygen intermediates [47], and the cytokine TGF- $\beta$ whose production correlates with diminished iNOS expression [48-52].

\section{Conclusion}

The data presented in this report suggest that resistance of human $L .(V$.) braziliensis isolates to $\mathrm{NO}$ is associated with nonresponsiveness to antimony therapy and an impaired macrophage activation to produce TNF- $\alpha$. Together with our previously published data indicating that NO resistance is associated with a worsened clinical presentation of American Tegumentary Leishmaniasis, these data suggest there may be consequences of $\mathrm{NO}$ resistance relevant to human disease. These observations may serve as a basis to test clinical isolates for resistance to toxic oxidants as a predictor of the course and drugresponsiveness of disease.

\section{Acknowledgements}

We are grateful to Elbe Myrtes Silva and Lúcia Reis for secretarial assistance. This work was supported by Instituto de Investigação em Imunologia (iii), NIH Tropical grants D43 TW007127-01 (RPA), US NIH grants Al045540 (MEW), Al067874 (MEW), Al059451 (MEW), R21 Al080801 (MEW), and P50 Al30693 (EMC, ARJ, RPA, MEW) and a Merit Review (MEW) and OEF-OIF grant (MEW) from the US Department of Veterans' Affairs and CNPq. AS, ARJ, EMC and RPA are funded by CNPq.

\section{Author details \\ 'Serviço de Imunologia, Hospital Universitário Prof. Edgard Santos, Universidade Federal da Bahia, Salvador, Bahia, Brazil. ${ }^{2}$ Universidade Federal de Sergipe, Aracaju, Sergipe, Brazil. ${ }^{3}$ Departments of Internal Medicine, Microbiology and Epidemiology, University of lowa and the VA Medical Center, lowa City, IA, USA.}

\section{Authors' contributions}

$A S, A G, J M B P, E M C$ and RPA participated equally in the study design. AS, AG, JMBP and RPA performed all the parasites experiments. AS, AG, ARJ, TRM, MEW and RPA drafted manuscript. AS, AG and JMBP participated in the experiments of human macrophages infection and cytokine detection. LHG ARJ, RPA and EMC participated of the procedure of parasite achievement and clinical exams at Corte de Pedra, Bahia, Brazil. All authors read and approved the final manuscript.

\section{Competing interests}

The authors declare that they have no competing interests.

Received: 5 November 2009 Accepted: 15 July 2010

Published: 15 July 2010 


\section{References}

1. Grimaldi G Jr, Tesh RB, McMahon-Pratt D: A review of the geographic distribution and epidemiology of leishmaniasis in the New World. Am J Trop Med Hyg 1989, 41:687-725.

2. Carvalho LP, Passos ST, Jesus AR: Imunopatogênese da Leishmaniose Tegumentar. Gaz Med Bahia 2005, 1:57-65.

3. Castes M, Trujillo D, Rojas ME, Fernandez CT, Araya L, Cabrera M, Blackwell J, Convit J: Serum levels of tumor necrosis factor in patients with American cutaneous leishmaniasis. Biol Res 1993, 26:233-238.

4. Carvalho EM, Barral A, Costa JM, Bittencourt A, Marsden P: Clinical and immunopathological aspects of disseminated cutaneous leishmaniasis. Acta Trop 1994, 56:315-325

5. Costa JM, Marsden PD, Llanos-Cuentas EA, Netto EM, Carvalho EM, Barral A, Rosa AC, Cuba CC, Magalhaes AV, Barreto AC: Disseminated cutaneous leishmaniasis in a field clinic in Bahia Brazil: a report of eight cases. $J$ Trop Med Hyg 1986, 89:319-323.

6. Alexander J, Russell DG: The interaction of Leishmania species with macrophages. Adv Parasitol 1992, 31:175-254.

7. Alexander J, Satoskar AR, Russell DG: Leishmania species: models of intracellular parasitism. J Cell Sci 1999, 112(18):2993-3002.

8. Liew FY, O'Donnell CA: Immunology of leishmaniasis. Adv Parasitol 1993, 32:161-259.

9. Trinchieri G, Gerosa F: Immunoregulation by interleukin-12. J Leukoc Biol 1996, 59:505-511.

10. Bogdan $C$, Rollinghoff $M$, Diefenbach $A$ : The role of nitric oxide in innate immunity. Immunol Rev 2000, 173:17-26.

11. Liew FY, Parkinson C, Millott S, Severn A, Carrier M: Tumour necrosis factor (TNF alpha) in leishmaniasis. I. TNF alpha mediates host protection against cutaneous leishmaniasis. Immunology 1990, 69:570-573.

12. Liew FY, Wei $X Q$, Proudfoot $L$ : Cytokines and nitric oxide as effector molecules against parasitic infections. Philos Trans $R$ Soc Lond B Biol Sci 1997, 352:1311-1315

13. Giudice A, Camada I, Leopoldo PT, Pereira JM, Riley LW, Wilson ME, Ho JL, de Jesus AR, Carvalho EM, Almeida RP: Resistance of Leishmania (Leishmania) amazonensis and Leishmania (Viannia) braziliensis to nitric oxide correlates with disease severity in Tegumentary Leishmaniasis. BMC Infect Dis 2007, 7:7.

14. Arevalo J, Ramirez L, Adaui V, Zimic M, Tulliano G, Miranda-Verastegui C, Lazo M, Loayza-Muro R, De Doncker S, Maurer A, et al: Influence of Leishmania (Viannia) species on the response to antimonial treatment in patients with American tegumentary leishmaniasis. J Infect Dis 2007, 195:1846-1851

15. Berman JD, Edwards N, King M, Grogl M: Biochemistry of Pentostam resistant Leishmania. Am J Trop Med Hyg 1989, 40:159-164

16. Berman JD, Gallalee JV, Best JM: Sodium stibogluconate (Pentostam) inhibition of glucose catabolism via the glycolytic pathway and fatty acid beta-oxidation in Leishmania mexicana amastigotes. Biochem Pharmacol 1987, 36:197-201.

17. Berman JD, Waddell D, Hanson BD: Biochemical mechanisms of the antileishmanial activity of sodium stibogluconate. Antimicrob Agents Chemother 1985, 27:916-920.

18. Holzmuller $P$, Sereno D, Lemesre JL: Lower nitric oxide susceptibility of trivalent antimony-resistant amastigotes of Leishmania infantum. Antimicrob Agents Chemother 2005, 49:4406-4409.

19. Sereno D, Holzmuller P, Mangot I, Cuny G, Ouaissi A, Lemesre JL: Antimonial-mediated DNA fragmentation in Leishmania infantum amastigotes. Antimicrob Agents Chemother 2001, 45:2064-2069.

20. Mookerjee Basu J, Mookerjee A, Sen P, Bhaumik S, Banerjee S, Naskar K, Choudhuri SK, Saha B, Raha S, Roy S: Sodium antimony gluconate induces generation of reactive oxygen species and nitric oxide via phosphoinositide 3-kinase and mitogen-activated protein kinase activation in Leishmania donovani-infected macrophages. Antimicrob Agents Chemother 2006, 50:1788-1797.

21. Croft SL, Sundar S, Fairlamb AH: Drug resistance in leishmaniasis. Clin Microbiol Rev 2006, 19:111-126.

22. Cupolillo E, Grimaldi G Jr, Momen H: A general classification of New World Leishmania using numerical zymotaxonomy. Am J Trop Med Hyg 1994, 50:296-311.

23. Miller MA, McGowan SE, Gantt KR, Champion M, Novick SL, Andersen KA, Bacchi CJ, Yarlett N, Britigan BE, Wilson ME: Inducible resistance to oxidant stress in the protozoan Leishmania chagasi. J Biol Chem 2000 275:33883-33889.

24. Wilson ME, Andersen KA, Britigan BE: Response of Leishmania chagasi promastigotes to oxidant stress. Infect Immunol 1994, 62:5133-5141.

25. Ettinger NA, Wilson ME: Macrophage and T-Cell Gene Expression in a Model of Early Infection with the Protozoan Leishmania chagasi. PLOS Negl Trop Dis 2008, 2:e252

26. Assreuy J, Cunha FQ, Epperlein M, Noronha-Dutra A, O'Donnell CA, Liew FY, Moncada S: Production of nitric oxide and superoxide by activated macrophages and killing of Leishmania major. Eur J Immunol 1994, 24:672-676.

27. Liew FY, Li Y, Moss D, Parkinson C, Rogers MV, Moncada S: Resistance to Leishmania major infection correlates with the induction of nitric oxide synthase in murine macrophages. Eur J Immunol 1991, 21:3009-3014.

28. Brandonisio O, Panaro MA, Fumarola I, Sisto M, Leogrande D, Acquafredda A, Spinelli R, Mitolo V: Macrophage chemotactic protein-1 and macrophage inflammatory protein-1 alpha induce nitric oxide release and enhance parasite killing in Leishmania infantum-infected human macrophages. Clin Exp Med 2002, 2:125-129.

29. Rich EA, Torres M, Sada E, Finegan CK, Hamilton BD, Toossi Z Mycobacterium tuberculosis (MTB)-stimulated production of nitric oxide by human alveolar macrophages and relationship of nitric oxide production to growth inhibition of MTB. Tuber Lung Dis 1997, 78:247-255.

30. Llanos-Cuentas A, Tulliano G, Araujo-Castillo R, Miranda-Verastegui C, Santamaria-Castrellon G, Ramirez L, Lazo M, De Doncker S, Boelaert M, Robays J, et al: Clinical and parasite species risk factors for pentavalent antimonial treatment failure in cutaneous leishmaniasis in Peru. Clin Infect Dis 2008, 46:223-231.

31. Machado P, Araujo C, Da Silva AT, Almeida RP, D'Oliveira A Jr, Bittencourt A Carvalho EM: Failure of early treatment of cutaneous leishmaniasis in preventing the development of an ulcer. Clin Infect Dis 2002, 34:E69-73.

32. Mauel J, Corradin SB, Buchmuller Rouiller Y: Nitrogen and oxygen metabolites and the killing of Leishmania by activated murine macrophages. Res Immunol 1991, 142:577-580, discussion 593-574.

33. Mauel J, Buchmuller-Rouiller Y: Effect of lipopolysaccharide on intracellular killing of Leishmania enriettii and correlation with macrophage oxidative metabolism. Eur J Immunol 1987, 17:203-208.

34. Channon JY, Roberts MB, Blackwell JM: A study of the differential respiratory burst activity elicited by promastigotes and amastigotes of Leishmania donovani in murine resident peritoneal macrophages. Immunology 1984, 53:345-355.

35. Murray HW: Cell-mediated immune response in experimental visceral leishmaniasis. II. Oxygen-dependent killing of intracellular Leishmania donovani amastigotes. J Immunol 1982, 129:351-357.

36. Zarley $\mathrm{JH}$, Britigan BE, Wilson ME: Hydrogen peroxide-mediated toxicity for Leishmania donovani chagasi promastigotes. Role of hydroxyl radical and protection by heat shock. J Clin Invest 1991, 88:1511-1521.

37. Iyer JP, Kaprakkaden A, Choudhary ML, Shaha C: Crucial role of cytosolic tryparedoxin peroxidase in Leishmania donovani survival drug response and virulence. Mol Microbiol 2008, 68:372-391.

38. Lemesre $J$, Sereno D, Daulouede S, Veyret B, Brajon N, Vincendeau P: Leishmania spp.: nitric oxide-mediated metabolic inhibition of promastigote and axenically grown amastigote forms. Exp Parasitol 1997, 86:58-68.

39. Iniesta V, Gomez-Nieto LC, Molano I, Mohedano A, Carcelen J, Miron C, Alonso C, Corraliza I: Arginase I induction in macrophages triggered by Th2-type cytokines supports the growth of intracellular Leishmania parasites. Parasite Immunol 2002, 24:113-118.

40. Roberts SC, Tancer MJ, Polinsky MR, Gibson KM, Heby O, Ullman B: Arginase plays a pivotal role in polyamine precursor metabolism in Leishmania. Characterization of gene deletion mutants. J Biol Chem 2004, 279:23668-23678.

41. Genestra M, Souza WJ, Guedes-Silva D, Machado GM, Cysne-Finkelstein L, Bezerra RJ, Monteiro F, Leon LL: Nitric oxide biosynthesis by Leishmania amazonensis promastigotes containing a high percentage of metacyclic forms. Arch Microbiol 2006, 185:348-354.

42. Gaur U, Showalter M, Hickerson S, Dalvi R, Turco SJ, Wilson ME, Beverley SM: Leishmania donovani lacking the Golgi GDP-Man transporter LPG2 exhibit attenuated virulence in mammalian hosts. Exp Parasitol 2009, 122:182-191. 
43. Carrera L, Gazzinelli RT, Badolato R, Hieny S, Muller W, Kuhn R, Sacks DL: Leishmania promastigotes selectively inhibit interleukin 12 induction in bone marrow-derived macrophages from susceptible and resistant mice. J Exp Med 1996, 183:515-526.

44. Bacellar O, Lessa H, Schriefer A, Machado P, de Jesus Ribeiro A, Dutra WO, Gollob KJ, Carvalho EM: Up-regulation of Th1-type responses in mucosal leishmaniasis patients. Infect Immun 2002, 70:6734-6740.

45. Olivier M, Gregory DJ, Forget G: Subversion mechanisms by which Leishmania parasites can escape the host immune response: a signaling point of view. Clin Microbiol Rev 2005, 18:293-305.

46. Padigel UM, Alexander J, Farrell JP: The role of interleukin-10 in susceptibility of BALB/c mice to infection with Leishmania mexicana and Leishmania amazonensis. J Immunol 2003, 171:3705-3710.

47. Belley A, Chadee K: Eicosanoid production by parasites: from pathogenesis to immunomodulation? Parasitol Today 1995, 11:327-334.

48. Bogdan C, Rollinghoff M: The immune response to Leishmania: mechanisms of parasite control and evasion. Int J Parasitol 1998, 28:121-134.

49. Gantt KR, Schultz-Cherry S, Rodriguez N, Jeronimo SM, Nascimento ET, Goldman TL, Recker TJ, Miller MA, Wilson ME: Activation of TGF-beta by Leishmania chagasi: importance for parasite survival in macrophages. $J$ Immunol 2003, 170:2613-2620.

50. Green SJ, Scheller LF, Marletta MA, Seguin MC, Klotz FW, Slayter M, Nelson BJ, Nacy CA: Nitric oxide: cytokine-regulation of nitric oxide in host resistance to intracellular pathogens. Immunol Lett 1994, 43:87-94

51. Nelson BJ, Ralph P, Green SJ, Nacy CA: Differential susceptibility of activated macrophage cytotoxic effector reactions to the suppressive effects of transforming growth factor-beta 1. J Immunol 1991, 146:1849-1857.

52. Stenger $\mathrm{S}$, Thuring $\mathrm{H}$, Rollinghoff $\mathrm{M}$, Bogdan $\mathrm{C}$ : Tissue expression of inducible nitric oxide synthase is closely associated with resistance to Leishmania major. J Exp Med 1994, 180:783-793.

\section{Pre-publication history}

The pre-publication history for this paper can be accessed here: http://www.biomedcentral.com/1471-2334/10/209/prepub

doi:10.1186/1471-2334-10-209

Cite this article as: Souza et al:: Resistance of Leishmania (Viannia) braziliensis to nitric oxide: correlation with antimony therapy and TNF- $\alpha$ production. BMC Infectious Diseases 2010 10:209.

\section{Submit your next manuscript to BioMed Central and take full advantage of:}

- Convenient online submission

- Thorough peer review

- No space constraints or color figure charges

- Immediate publication on acceptance

- Inclusion in PubMed, CAS, Scopus and Google Scholar

- Research which is freely available for redistribution 